\title{
Layout Volumes of the Hypercube
}

\author{
Lubomir Torok ${ }^{1}$ and Imrich Vrt'o ${ }^{2}$ \\ 1 Institute of Mathematics and Computer Science, \\ Severná 5, 97401 Banská Bystrica, Slovak Republic \\ 2 Institute of Mathematics, Slovak Academy of Sciences, \\ Dúbravská 9, 84104 Bratislava, Slovak Republic
}

\begin{abstract}
We study 3-dimensional layouts of the hypercube in a 1active layer and a general model. The problem can be understood as a graph drawing problem in $3 D$ space and was addressed at Graph Drawing 2003 [5]. For both models we prove general lower bounds which relate volumes of layouts to a graph parameter called cutwidth. Then we propose tight bounds on volumes of layouts of $N$-vertex hypercubes. Especially, we have $\mathrm{VOL}_{1-A L}\left(Q_{\log N}\right)=\frac{2}{3} N^{\frac{3}{2}} \log N+O\left(N^{\frac{3}{2}}\right)$, for even $\log N$ and $\operatorname{VOL}\left(Q_{\log N}\right)=\frac{2 \sqrt{6}}{9} N^{\frac{3}{2}}+O\left(N^{4 / 3} \log N\right)$, for $\log N$ divisible by 3 . The 1-active layer layout can be easily extended to a 2-active layer (bottom and top) layout which improves a result from [5].
\end{abstract}

\section{Introduction}

The research on three-dimensional circuit layouts started in seminal works [15, 17] as a response to advances in VLSI technology. Their model of a 3-dimensional circuit was a natural generalization of the 2-dimensional model [18]. Several basic results have been proved since then which show that the 3-dimensional layout may essentially reduce material, measured as volume $[6,12]$. The problem may be also understood as a special 3-dimensional orthogonal drawing of graphs, see e.g., [9]. In all these models the degrees of vertices of a graph, which represents the circuit, are at most 6 . There exist only a few papers investigating layouts of graphs of arbitrary degrees, e.g., $[4,5]$. There are two basic variants of the layout model. In a 1-active layer model, a vertex of degree $d$ is represented by a square of side $d$ and is placed in the bottom layer of the 3-dimensional grid. In the general model, a vertex of degree $d$ is represented by a cube of side $d$ and can lie anywhere in the grid. In both models edges are routed as edge disjoint paths in the underlying grid. The variants are straightforward extensions of linear layouts [13] and 2-dimensional layouts [18,19], respectively, and generalizations of 3-dimensional layouts for bounded degree graphs mentioned above.

In this paper we solve an open problem posed by Calamoneri and Massini [5] concerning the layout volume of the hypercube. We solve the problem for both models. First we prove general lower bounds on volumes of any graph $G$ of cutwidth $\mathrm{cw}(G)$ :

* This research was partially supported by the VEGA grant No. 2/3164/23. 


$$
\begin{gathered}
\operatorname{VOL}_{1-A L}(G) \geq \operatorname{cw}(G) \sqrt{\sum_{v \in V} \operatorname{deg}^{2}(v)}, \\
\operatorname{VOL}(G) \geq(\operatorname{cw}(G)-\sqrt{2 \mathrm{cw}(G)})^{\frac{3}{2}} .
\end{gathered}
$$

The lower bounds represent 3-dimensional analogues of similar estimations of the area of linear or 2-dimensional layouts $[13,16]$ and are of special interest. Then we propose optimal layouts in both models for the $\log N$-dimensional hypercube. Especially, for even $\log N$ we have

$$
\begin{gathered}
\operatorname{VOL}_{1-A L}\left(Q_{\log N}\right)=\frac{2}{3} N^{\frac{3}{2}} \log N+O\left(N^{\frac{3}{2}}\right), \\
\operatorname{VOL}\left(Q_{\log N}\right)=\frac{2 \sqrt{6}}{9} N^{\frac{3}{2}}+O\left(N^{\frac{4}{3}} \log N\right),
\end{gathered}
$$

for $\log N$ divisible by 3 . Recall that 2-dimensional layouts of hypercubes were studied in several papers $[3,8,11,14]$, but only recently an asymptotically exact result has appeared [10], for even $\log N$.

Our layout can be easily extended to the 2-active layer model, where vertices represented as rectangles are placed on the bottom and top layer of a grid. For such a model, Calamoneri and Massini [5] designed nearly optimal layout for the hypercube. We prove an optimal layout for the hypercube in this model.

\section{Preliminaries}

The 3-dimensional grid consists of all points $(a, b, c)$ of integer coordinates in the standard $x y z$-coordinate system. Two points are joined by a straightline segment if, and only if, their Euclidean distance is 1 . The lines parallel to axes and going through the points are called tracks. At the same time we consider the 3-dimensional grid as an infinite grid graph whose vertices corresponds to points and edges are the segments of lengths 1 . The 3-dimensional 1-active layer layout of a graph $G$ is a mapping of $G$ into the grid such the following conditions are satisfied:

- A vertex of degree $d$ is represented by a square of integer coordinates of side $d$ lying in the basic plane given by $z=0$. The sides of the square are parallel to the $x$ and $y$ axes.

- Two vertices (squares) do not touch.

- Edges are represented as edge disjoint paths in the grid graph in the halfspace above the basic plane. A path touches only two squares which represent the endvertices of the corresponding edge.

The 3-dimensional general layout of a graph $G$ is a mapping of $G$ into the grid such the following conditions are satisfied:

- A vertex of degree $d$ is represented by a cube of integer coordinates of side edge length $d$. The edges of the cube are parallel to the axes.

- Two vertices (cubes) do not touch. 
- Edges are represented as edge disjoint paths in the grid graph. A path touches only two cubes which represent the endvertices of the corresponding graph edge.

The volume of the 3-dimensional 1-active layer (general) layout of $G$, denoted by $\operatorname{VOL}_{1-A L}(G)$ (denoted by $\operatorname{VOL}(G)$ ) is defined as the volume of the smallest box containing the layout. From technical reasons we assume that the coordinates of the box vertices are of the form $(a+1 / 2, b+1 / 2, c+1 / 2)$, for some integers $a, b, c$.

Remark. The models we described, differs from the so called multilayer model [7], in which vertices are represented as boxes lying on the bottom layer and edges are routed in layers parallel to the basic plane.

Let $\phi: V \rightarrow 1,2,3, \ldots,|V|$ be a 1-1 labelling of vertices of a graph $G=(V, E)$. Define

$$
\operatorname{cw}(G, \phi)=\max _{i}\{|\{u v \in E: \phi(u) \leq i<\phi(v)\}|\} .
$$

The cutwidth of the graph $G$ is defined as

$$
\mathrm{cw}(G)=\min _{\phi}\{\mathrm{cw}(G, \phi)\} .
$$

The cutwidth is strongly related to linear layouts and, roughly saying, represents the largest edge cut in a graph which is embedded in a line.

Let $\operatorname{deg}(v)$ denote the degree of a vertex $v$. If $N$ is a power of two, the $\log N$ dimensional hypercube graph has a vertex set consisting of all binary strings of length $\log N$. Two vertices are joined by an edge if the corresponding strings differ in precisely one position.

\section{Lower Bounds}

In this section we prove a lower bound which is of special interest for its general use and easy applicability.

Theorem 1. The volume of the 3-dimensional 1-active layer layout of any graph $G$ of cutwidth $\mathrm{cw}(G)$ satisfies

$$
\operatorname{VOL}_{1-A L}(G) \geq \mathrm{cw}(G) \sqrt{\sum_{v \in V} \operatorname{deg}^{2}(v)} .
$$

Proof. Assume we have an optimal 1-active layer layout of $G$ of the volume $\mathrm{VOL}_{1-A L}(G)$. The layout is put into a box of the width $W$, length $L$ and height $H$, where $W, L, H$ are measured along the $x, y, z$ axes, respectively. Thus

$$
\operatorname{VOL}_{1-A L}(G)=W L H .
$$

As two vertices do not touch we have

$$
W L \geq \sum_{v \in V}(\operatorname{deg}(v)+1)^{2}
$$



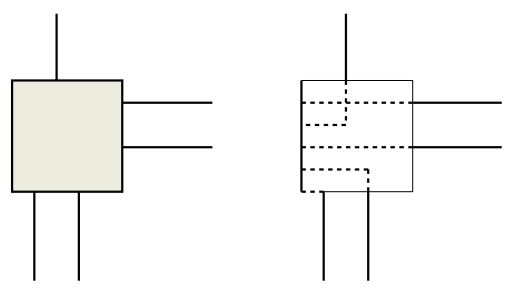

Fig. 1. Replacing a $5 \times 5$ square vertex by a straight-line segment.

Consider now the first layer. For every vertex (square) remove its sides except for the left one. It is easy to see that the edges which ended on those 3 sides can be prolonged and connected to the left side using the original tracks and such that the edges are edge disjoint. See Fig. 1

We obtain a new layout in which the square vertices are replaced by straightline segments. Now we use a similar idea as in earlier paper [16] (Lemma 2.1), for estimating the area of 2-dimensional layouts. For the sake of completeness we repeat the argument. Any such segment is identified by its coordinates given by the position of the lower end of the segment. Sort the segments according to their coordinates lexicographically. Label the segments by $1,2, \ldots,|V|$, according to the lexicographic order. Let $\phi$ denote this labelling. Take the first $i$ segments in this labelling. We can find a surface, normal to $x y$-plane, which separates precisely $i$ segments from the rest, as in Fig. 2.

The area of the surface is $(L+1) H$. Clearly the number of edges between the two parts of the layout is given by

$$
\{|\{u v \in E: \phi(u) \leq i<\phi(v)\}|\} \leq(L+1) H
$$

Maximizing the left hand side over all $i$ 's and then minimizing it over all $\phi$ 's we get the cutwidth of $G$ on the left side. By rearranging the terms we have

$$
(L+1) H \geq \operatorname{cw}(G) .
$$

We may repeat the above argument by changing the role of $L$ and $W$. We get

$$
(W+1) H \geq \operatorname{cw}(G) .
$$

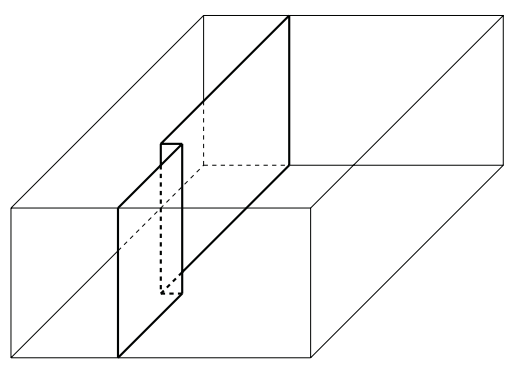

Fig. 2. A 3D cut of the volume. 
Combining relations (1),(2),(4) and (3) we have

$$
\begin{aligned}
\mathrm{VOL}_{1-A L}(G) & \geq(\operatorname{cw}(G)-H) \sqrt{\sum_{v \in G}(\operatorname{deg}(v)+1)^{2}} \\
& =\left(\operatorname{cw}(G)-\frac{\mathrm{VOL}_{1-A L}(G)}{W L}\right) \sqrt{\sum_{v \in G}(\operatorname{deg}(v)+1)^{2}} \\
& \geq\left(\operatorname{cw}(G)-\frac{\mathrm{VOL}_{1-A L}(G)}{\sum_{v \in G}(\operatorname{deg}(v)+1)^{2}}\right) \sqrt{\sum_{v \in G}(\operatorname{deg}(v)+1)^{2}} \\
& \geq \operatorname{cw}(G) \sqrt{\sum_{v \in G}(\operatorname{deg}(v)+1)^{2}}-\frac{\operatorname{VOL}_{1-A L}(G)}{\sqrt{\sum_{v \in G}(\operatorname{deg}(v)+1)^{2}}} .
\end{aligned}
$$

By eliminating $\mathrm{VOL}_{1-A L}(G)$ and some algebraic manipulations we get

$$
\operatorname{VOL}_{1-A L}(G) \geq \operatorname{cw}(G)\left(\sqrt{\sum_{v \in V}(\operatorname{deg}(v)+1)^{2}}-1\right) \geq \operatorname{cw}(G) \sqrt{\sum_{v \in V} \operatorname{deg}^{2}(v)} .
$$

Remark. The idea of cutting of the 3-dimensional layout into special parts was used in [6] in a model for bounded degree graphs, where they considered the so called special bisection width of a graph which is however in general a smaller quantity than the cutwidth.

Corollary 1. The optimal volume of the 3-dimensional 1-active layer layout of the $N$-vertex hypercube $Q_{\log N}$ satisfies

$$
\mathrm{VOL}_{1-A L}\left(Q_{\log N}\right) \geq \frac{2}{3} N^{\frac{3}{2}} \log N+O(\sqrt{N} \log N) .
$$

Proof. Several papers proved that $\mathrm{cw}\left(Q_{\log N}\right)=\lfloor 2 N / 3\rfloor$, e.g., [1,2].

Theorem 2. The optimal volume of the 3-dimensional layout of any graph $G=$ $(V, E)$ satisfies

$$
\operatorname{VOL}(G) \geq(\operatorname{cw}(G)-\sqrt{2 \mathrm{cw}(G)})^{\frac{3}{2}} .
$$

Proof. Consider an optimal 3-dimensional layout of $G$. Assume the layout is put into a box of sizes $W, L, H$ such that

$$
W \geq L \geq H
$$

Take any vertex (a cube of sizes $n \times n \times n$ ) and choose one of its vertical "edges" (a straightline segment). Delete the cube except for that segment. Observe that the graph edges originally attached to the cube can be prolonged and connected to the segment using edge disjoint paths along the tracks. Repeating this operation for all vertices we get a new layout in which the vertices are replaced by segments of length $n$ and edges are routed in the edge disjoint manner. Similarly as in the proof of Theorem 1, we can find a surface, see Fig. 3, which 


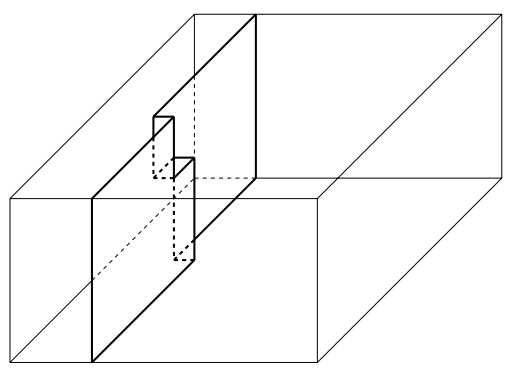

Fig. 3. A $3 \mathrm{D}$ cut of the volume.

separates the segments into two parts such that there are at least $\mathrm{cw}(G)$ edges between the segments.

The edges must cross the surface. The area of the surface is $H L+H+1$. It follows

$$
H L+H+1 \geq \operatorname{cw}(G)
$$

Then

$$
H L \geq \mathrm{cw}(G)-1-H .
$$

The relation (5) implies

$$
\begin{aligned}
& L W \geq \mathrm{cw}(G)-1-H . \\
& W H \geq \mathrm{cw}(G)-1-H .
\end{aligned}
$$

Multiplying (6)(7) and (8) and taking the cube root we have

$$
\operatorname{VOL}^{\frac{2}{3}}(G) \geq \mathrm{cw}(G)-1-H \geq \mathrm{cw}(G)-1-\operatorname{VOL}^{\frac{1}{3}}(G) .
$$

Solving this quadratic inequality for $\operatorname{VOL}^{\frac{1}{3}}(G)$ we get the lower bound.

Corollary 2. The optimal volume of the 3-dimensional layout of the $N$-vertex hypercube $Q_{\log N}$ satisfies

$$
\operatorname{VOL}\left(Q_{\log N}\right) \geq \frac{2 \sqrt{6}}{9} N^{\frac{3}{2}}+O(N) .
$$

\section{One-Active Layer Layout of Hypercubes}

Theorem 3. The optimal volume of the 1-active layer layout of the $\log N$ dimensional hypercube satisfies

$$
\mathrm{VOL}_{1-A L}\left(Q_{\log N}\right) \leq \frac{2^{\frac{i}{2}+1}}{3} N^{\frac{3}{2}} \log N+O\left(N^{\frac{3}{2}}\right),
$$

where $i=\log (N \bmod 2)$. 


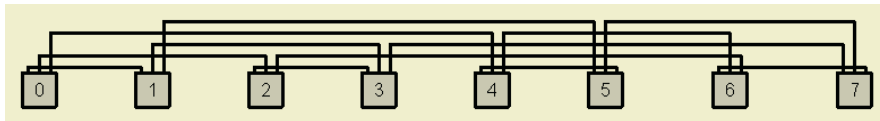

Fig. 4. Linear layout of $Q_{3}$.

Proof. Assume $\log N$ is divisible by 2. The second case is similar. Our basic building block is a linear layout of the $m$-dimensional hypercube $Q_{m}$ using $\mathrm{cw}\left(Q_{m}\right)=\left\lfloor 2^{m+1} / 3\right\rfloor$ horizontal tracks, while every vertex is represented by a square of size $m$. See Figure 4 for the case $m=3$. Such a layout can be obtained by placing the vertices on the line in the natural order and assigning the edges to tracks properly. This was observed in several papers, e.g., [8,10,11]. We utilize a fact that $Q_{\log N}$ can be represented as a cartesian product $Q_{\frac{\log N}{2}} \times Q_{\frac{\log N}{2}}$. Using the linear layout minimizing the cutwidth for $Q_{\frac{\log N}{2}}$, one can easily design a 2-dimensional layout of $Q_{\log N}$ as shown in Figure 5 , for $\mathrm{N}=64$.

Now rotating every edge in the angle $\pi / 2$ around the line defined by its endpoints and compacting the vertices in the natural way we get a 1-active layer layout of the volume

$$
\left\lfloor\frac{2}{3} N^{\frac{1}{2}}\right\rfloor \times N(\log N+1)^{2} .
$$

The first term stands for the height of the layout and the second one for the occupied area on the 1st layer. We decrease the height of the layout by a factor of $\log N$ in the following way. Consider the layout of any $Q_{\frac{\log N}{2}}$ - the sublayout of the global layout of $Q_{\log N}$. Assume the vertices of $Q_{\frac{\log N}{2}}$ are aligned along

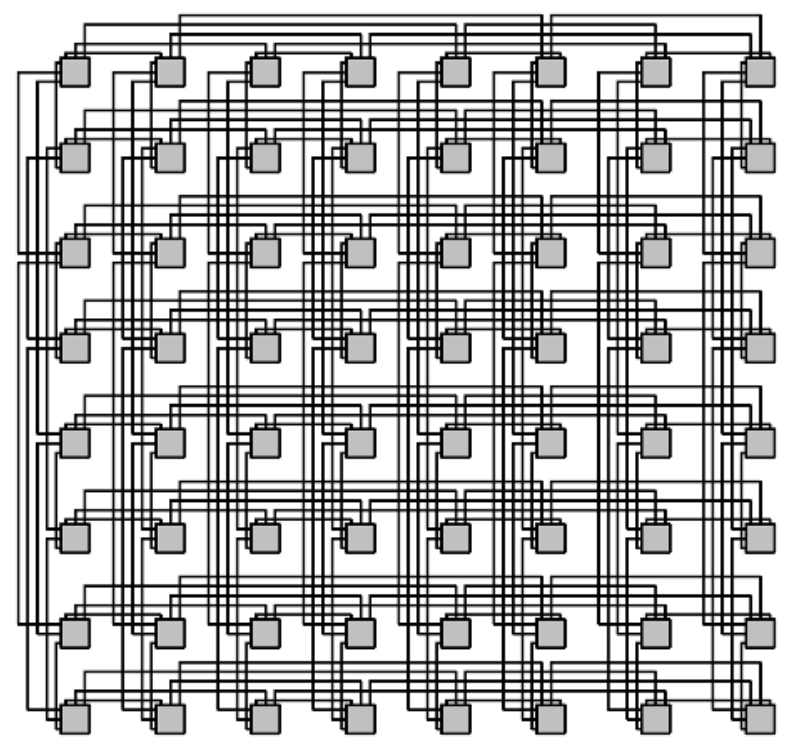

Fig. 5. 2-dimensional layout of $Q_{6}=Q_{3} \times Q_{3}$. 
the $x$-axis. The edges occupy $\left\lfloor 2 N^{1 / 2} / 3\right\rfloor$ tracks parallel to $x$. Divide the edges evenly into $\log N$ groups according to the distance of the corresponding tracks from the basic plane. I.e., the first group occupy the first $t=\left\lceil\left\lfloor 2 N^{1 / 2} / 3\right\rfloor / \log N\right\rceil$ tracks when counting from the basic plane, the second group occupy the second $t$ tracks, and so on. Reattach the edges to their corresponding vertices such that:

i) The endpoints of edges of the same group has the same $y$ coordinate.

ii) The endpoints of edges of different groups have different $y$ coordinate.

iii) The endpoints of edges lies in the first or the third "quadrant" of the square vertex. This can be viewed as a "shifting" of edges in $y$ direction on a proper place on its square endvertices. Finally, decrease the height $h$ of every track to $(h \bmod t)+1$ and reroute correspondigly the edges. See Figure 6 .
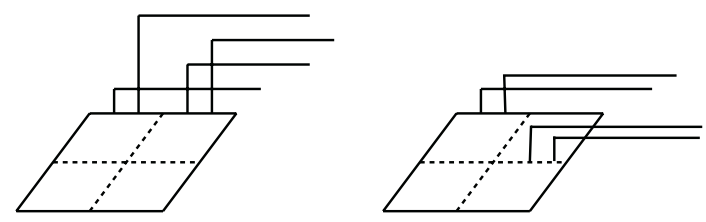

Fig. 6. Decreasing the height of the layout.

We repeat this operation for all $Q_{\frac{\log N}{2}}$ 's in $x$ and $y$ directions. For the $y$ direction, the endpoits of edges lie in the second and fourth quadrants of square vertices. This avoids overlapping of vertical parts of two edges starting in the same square vertex. The new height of the layout is $t$, which implies the claimed volume.

\section{Layouts of Hypercubes in the General Model}

Theorem 4. The optimal volume of the general 3-dimensional layout of the $\log N$-dimensional hypercube satisfies

$$
\operatorname{VOL}\left(Q_{\log N}\right) \leq \frac{2^{\frac{i}{2}+1} \sqrt{6}}{9} N^{\frac{3}{2}}+O\left(N^{\frac{4}{3}} \log N\right),
$$

where $i=\log (N \bmod 3)$.

Proof. Let $\log N$ be divisible by 3 . The other 2 cases are similar. Consider a cube of edge length $n=\log N$ in the $3 D$ grid. Consider another cube of edge length $s+n$ such that the first cube is positioned "centrally" in the second one and

$$
\left.s=\sqrt{\left\lfloor\frac{2 N^{\frac{1}{3}}}{3}\right\rfloor}\right\rceil \text {. }
$$

Place $N^{1 / 3}$ copies of the second cube (with the first one inside) along the $x$ axis with unit spacing such that they form a box $C$ of size $(s+n+1) N^{1 / 3} \times(s+n) \times$ $(s+n)$. Note that in the box $\mathrm{C}$, there are at least 


$$
(s+n)^{2}-n^{2} \geq\left\lfloor\frac{2 N^{\frac{1}{3}}}{3}\right\rfloor
$$

tracks, parallel to the $x$-axis, which do not cross the cubes of sides $n$. Now let the $N^{1 / 3}$ small cubes be vertices of a $Q_{\frac{\log N}{3}}$ placed along the $x$-axis in the natural order. Thus having cutwidth $\left\lfloor 2 N^{1 / 3} / 3\right\rfloor$, if we assume that the edges are drawn as in the linear layout in one plane. It is easy to redraw the edges of $Q_{\frac{\log N}{3}}$ such that they lie in the box $C$ and if two edges shared the same track in the linear layout they will share the same track in $C$. Moreover the edges are attached to the opposite sides of a vertex only. See Figure 7.

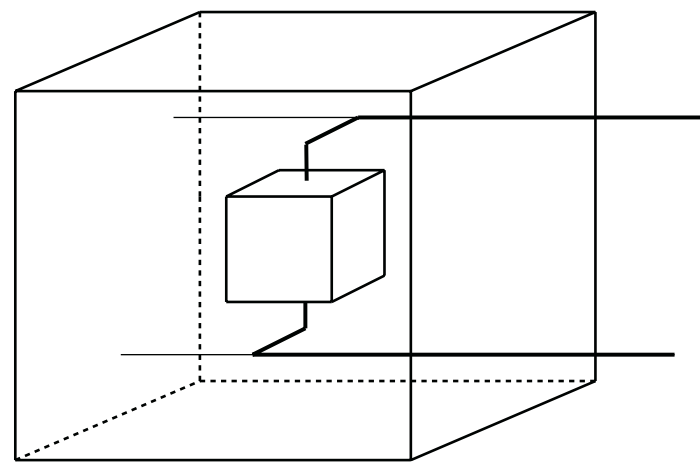

Fig. 7. Routing edges in the general model.

Finally, we use again the fact that $Q_{\log N}=Q_{\frac{\log N}{3}} \times Q_{\frac{\log N}{3}} \times Q_{\frac{\log N}{3}}$. Repeating the above construction for all $Q_{\frac{\log N}{3}}$ 's in all 3 dimensions we get a layout for $Q_{\log N}$. One can check that the layout satisfies the assumptions of the model. The total volume is

$$
(s+n+1)^{3} N=\frac{2 \sqrt{6}}{9} N^{\frac{3}{2}}+O\left(N^{\frac{4}{3}} \log N\right) .
$$

\section{A 2-Active Layers Layout for Hypercubes}

Calamoneri and Massini [5] proposed a 2-active layer layout model for bipartite graphs. In this model, all vertices are represented as rectangles and lie on two opposite sides of the bounding box of the layout volume. The vertices are distributed evenly between the two layers. The other properties of the model are as in the 1-active layer layout model. Particularly, they studied layouts of $\log N$-dimensional hypercubes, assuming that each vertex is represented as a $1 \times\left\lceil\frac{\log N}{2}-1\right\rceil$ rectangle, and proved

$$
\begin{aligned}
& \operatorname{VOL}_{2-A L}\left(Q_{\log N}\right)=\Omega\left(N^{\frac{3}{2}} \log \frac{1}{2} N\right), \\
& \operatorname{VOL}_{2-A L}\left(Q_{\log N}\right)=O\left(N^{\frac{3}{2}} \log N\right) .
\end{aligned}
$$


We follow the same model but we represent every vertex of the hypercube as a square of side $\log N$. We have

Theorem 5. The optimal volume of the 3-dimensional 2-active layer layout of the $N$-vertex hypercube $Q_{\log N}$ satisfies

$$
\operatorname{VOL}_{2-A L}\left(Q_{\log N}\right)=\Theta\left(N^{\frac{3}{2}} \log N\right)
$$

Proof. The lower bound is proved in a similar way as in Theorem 1 . The matching upper bound is obtained by placing two $(\log N-1)$-dimensional hypercubes on the two opposite layers using the 1-active layout from Theorem 3 and adding the edges of the $\log N$-th dimension as straight-line segments between the layers.

\section{References}

1. Bel Hala, A., Congestion optimale du plongement de l'hypercube $H(n)$ dans la chaine $P(2 n)$, ITA 27 (1993), 465-481.

2. Bezrukov, S., Chavez, J.D., Harper, L.H., Röttger, M., Schroeder, U.-P., The congestion of $n$-cube layout on a rectangular grid, Discrete Mathematics, 213, (2000), 13-19.

3. Brebner, G., Relating routing graphs and two-dimensional grids, in: Proc. VLSI: Algorithms and Architectures, North Holland, 1985.

4. Biedl, T., Thiele, T., Wood, D.R., Three-dimensional orthogonal graph drawing with optimal volume, in: Proc. 11th Intl. Symposium on Graph Drawing, Lecture Notes in Computer Science 1984, Springer, Berlin, 2000, 284-295.

5. Calamoneri, T., Massini, A., Nearly optimal three-dimensional layout of hypercube networks, in: Proc. 11th Intl. Symposium on Graph Drawing, Lecture Notes in Computer Science 2912, Springer, 2003, 247-258.

6. Calamoneri, T., Massini, A., Optimal three-dimensional layout of interconnection networks, Theoretical Computer Science 255 (2001), 263-279.

7. Chi-Hsiang Yeh, Varvarigos, E.A., Parhami, B., Multilayer VLSI layout for interconnection networks, in: Proc. Intl. Conf. on Parallel Processing, 2000, 21-24.

8. Chi-Hsiang Yeh, Varvarigos, E.A., Parhami, B., Efficient VLSI Layouts of hypercubic networks, in: Proc. Frontiers of Massivelly Parallel Computation, 1999, 98-105.

9. Eades, P., Symvonis, A., Whitesides, S., Two algorithms for three-dimensional graph drawing, in: Proc. 4th Intl. Symposium on Graph Drawing, Lecture Notes in Computer Science 1190, Springer Berlin 1996, 139-154.

10. Even, S., Kupershtok, R., Layout area of the hypercube, Journal of Interconnection Networks 4 (2003), 395-417.

11. Greenberg, R.I., Lee Guan, On the area of hypercube networks, Information Processing Letters 41 (2002), 41-46.

12. Leighton, F.T., Rosenberg, A.L., Three-dimensional circuit layouts, SIAM Journal on Computing 15 (1986), 793-813.

13. Leiserson, C.E., Area-efficient graph layouts (for VLSI), in: Proc. 21st Annual IEEE Symposium on Foundation of Computer Science, IEEE Computer Science Press, 1980, 270-281

14. Patel, A., Kusalik, A, McCroskey, C., Area-efficient layouts for binary hypercubes, IEEE Transactions on Computers 49 (2000), 160-169. 
15. Preparata, F.P., Optimal three-dimensional VLSI layouts, Mathematical Systems Theory 16 (1983), 1-8.

16. Raspaud, A., Sýkora, O., Vrt'o, I., Cutwidth of the de Bruijn graph, RAIRO 26 (1995), 509-514.

17. Rosenberg, A.L., Three-dimensional VLSI: A case study, Journal of the ACM 30 (1983), 397-416.

18. Thompson, C.D., Area-time complexity for VLSI, in: Proc. 11th Annual ACM Symposium on Theory of Computing, 1979, 81-88.

19. Ullman, J.D., Computational Aspects of VLSI, Comp. Sci. Press, Rockville, 1983. 